MATEC Web of Conferences 39, 02004 (2016)

DOI: $10.1051 /$ matecconf/20163902004

(c) Owned by the authors, published by EDP Sciences, 2016

\title{
Recognition method of metal fracture images based on Wavelet kurtosis and Relevance vector machine
}

\author{
Yi SUN ${ }^{1}$, Zhinong $\mathrm{LI}^{1, a}$, Jingwen YAN ${ }^{2}$ \\ ${ }^{1}$ Key Laboratory of Nondestructive Testing, Ministry of Education, Nanchang Hangkong University, Nanchang, China \\ ${ }^{2}$ Colleage of Engineering, Shantou University, Shantou, China
}

\begin{abstract}
The useful information extracted from fracture images is the most fundamental problem of quantitative analysis and intelligent diagnosis of metal fracture. The pattern recognition or classification is the critical issue of failure analysis of metal fracture. In this paper, combining wavelet transform, kurtosis with relevance vector machine (RVM), a new recognition method based on wavelet kurtosis and RVM, which is named wavelet kurtosis-RVM, is proposed. In the proposed method, wavelet kurtosis is used as a feature vector, and RVM as a classifier. The proposed method has been successfully applied to the recognition of fracture images. The proposed method is also compared with the wavelet entropy-RVM recognition method and wavelet kurtosis-SVM recognition method. The experiment result shows that the proposed method is very effective. Compared with the Wavelet entropy-RVM recognition method, Wavelet kurtosis is more sensitive to the texture change of metal fracture and suitable for feature extraction of metal fracture. Compared with the Wavelet kurtosis-SVM recognition method, The proposed method and Wavelet kurtosis-SVM recognition method have the same good recognition rate. However, in the recognition speed, the Wavelet kurtosis-RVM recognition method is obviously superior to the Wavelet kurtosis-SVM recognition method, especially in the increase of training samples.
\end{abstract}

Keywords: Wavelet transform, Kurtosis, Relevance vector machine (RVM), Feature extraction, Pattern recognition, Metal fracture, Wavelet entropy, Support vector machine (SVM).

\section{INTRODUCTION}

In the many failure analysis, fracture analysis is a very important technology. Fracture is formed through the different stage, i.e. formation, expansion and final break [1]. When the fracture occurs, some corresponding marks, morphology and characteristics will remain on the fracture surface. We analyze these fractures mainly through observing, identifying and analyzing these marks, morphology and characteristics, try to reveal some factors related with the fracture process, and determine the failure mechanism of fracture. Therefore, fracture analysis is very important in the fracture failure analysis.

Pattern recognition or classification of fracture image is a critical issue to diagnose fracture failure. At present, many feature extraction and recognition methods have been proposed. However, in these traditional recognition methods, the main method is wavelet transform [2-5]. YAN [2] proposed a nonlinear pattern recognition method based on wavelet transform and neural network. Kenta [3] proposed a detecting method of striation region of fatigue fracture surface using the wavelet transform, the experiment result verified the effectiveness of the proposed method. XU [4] studies the feature extraction method of metal fracture image based on wavelet transform. ZHANG [5] proposed a recognition method based on tree structure wavelet transform. In the proposed method, standard deviations of each wavelet transform frequency band output is used as the characteristics of fracture image recognition, minimal distance classifier is used to classify the fracture image. However, in these recognition methods of metal fracture image based on wavelet transform, different feature vector and different classifier usually leads to the obvious difference of recognition rate. Therefore, how to effectively extract the fracture feature using wavelet transform and classifier selection become two key problems of this method.

Kurtosis is a normalized time-domain statistics. Compared with the traditional feature vector of fracture image, such as L1 norm and energy etc, Kurtosis is more sensitive to the texture feature. In additional, kurtosis is also sensitive to the singularity, and not sensitive to the direction. Therefore, in this paper, combining wavelet transform with kurtosis, a new concept, which is named wavelet kurtosis, is proposed and used to extract the texture features of metal fracture image. Meanwhile, the proposed wavelet kurtosis is compared with wavelet entropy in the feature of metal fracture image.

Relevance vector machine (RVM), which is proposed by Tipping[6], is a probabilistic sparse kernel model identical in functional form to the SVM. A Bayesian

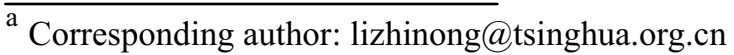


approach is adopted in the machine learning. The most compelling feature of the RVM is that, while capable of generalization performance comparable to an equivalent SVM, it typically utilizes dramatically fewer kernel functions. Furthermore, the RVM suffers from none of limitations of the SVM. When the RVM is applied to the classification problem, it can give a probability measurement.

Here, combining wavelet kurtosis with the RVM, a recognition method of metal fracture image based on wavelet kurtosis-RVM is proposed. At the same time, the proposed method is compared with the wavelet entropyRVM and wavelet kurtosis-SVM recognition method. The experiment result verifies the validity of the proposed method.

\section{Feature extraction method based on wavelet kurtosis}

Wavelet transform has good prospect in image processing because of good local characteristic [7]. In the recognition method of metal fracture image based on wavelet transform, the wavelet coefficients is always combined with information entropy, the energy distribution in each scale of wavelet is used to reflect the texture feature of fracture image, this defined entropy is called wavelet entropy[8], which is shown as follows

$$
H_{W T}=-\sum_{i=1}^{m} p_{i} \log _{2}{ }^{p_{i}}
$$

Where $p_{i}$ is the relative wavelet energy, which stands for the energy ratio of the i-th decomposition level and all decomposition levels. The wavelet entropy HWT can reflect the information amount of image, the larger HWT is, the more abundant detail component of image contains. At present, the wavelet entropy has become a main feature extraction method of image [9-11].

However, when wavelet entropy is used to extract the feature of fracture image, it reflects the differences of energy distribution in each decomposition scale, and cannot clearly reflect the difference of texture details in fracture image. Therefore, we should try to look for new feature extraction method to improve the recognition rate of metal fracture image.

Based on this, in this paper, a new feature extraction method of metal fracture image based on wavelet kurtosis is proposed. Kurtosis, which is a normalized fourth-order central moment, can be used to describe the mutation intensity of signal. Here, combining kurtosis with wavelet transform, a new concept of wavelet kurtosis is proposed and used to extract feature of metal fracture image. The define of wavelet kurtosis is as follows

$$
K_{W T}=\frac{1}{M \times N} \sum_{i=1}^{M} \sum_{j=1}^{N}\left(\frac{x(i, j)-\bar{x}}{\sigma}\right)^{4}
$$

Where is the size of fracture image, the symbol $i, j$ is respectively the row and column of subband; the symbol $x$ is a wavelet coefficient of this subband, $\bar{x}$ is a mean of wavelet coefficients, $\sigma$ is a standard deviation, which is calculated as follows

$$
\sigma=\sqrt{\frac{1}{M N} \sum_{i=1}^{M} \sum_{j=1}^{N}(x(i, j)-\bar{x})^{2}}
$$

From Eq.(1) and Eq. (2), the wavelet entropy reflects the difference of energy distribution in fracture image. Because the wavelet entropy is proportional to the square of wavelet coefficient, wavelet kurtosis is proportional to the fourth power of wavelet coefficient. Therefore wavelet kurtosis is more sensitive to the texture change of metal fracture image than wavelet entropy. Compared with the wavelet entropy, the wavelet kurtosis can be more effective to reflect small change of texture feature, so the wavelet kurtosis is more suitable to extract the feature of metal fracture image.

\section{Wavelet kurtosis-RVM Recognition method}

The proposed wavelet kurtosis-RVM recognition method is as shown in Fig.1. From Fig.1, the proposed method consists of two important process, one is the feature extraction of fracture image, another is the selection of recognizer. Eq.(2) is used to calculate eigenvectors of each fracture image. For the classifier, we adopt the relevance vector machine (RVM) proposed by Tipping[6]. The RVM is a probabilistic sparse kernel model identical in functional form to the SVM, as with the SVM, the nonlinear problem in the low-dimensional space can be mapped into a linear problem in the highdimensional space by kernel function. At present, the RVM has been widely applied to the recognition field.

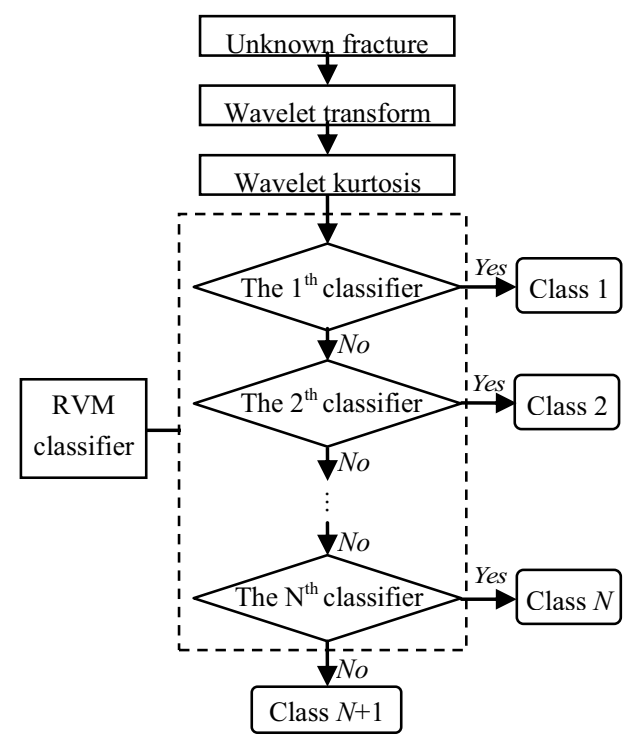

Fig.1 Wavelet kurtosis -RVM recognition method

Assume $\left\{x_{i}, t_{i}\right\}_{i=1}^{N}$ is a training sample, $x_{i}$ is a characteristic data to be classified, $t_{i} \in\{0,1\}$ is a output target of recognition, then we can obtain the predictive function 


$$
y(x, w)=\sum_{i=1}^{M} w_{i} K\left(x, x_{i}\right)+w_{0}
$$

where $M$ is the number of kernel function, $K\left(x, x_{i}\right)$ is a kernel function, $\left\{w_{i}\right\}$ is a weight of relevance vector, $w_{0}$ is an initial weight.

Relevance vector machine is used to reduce the above weights under the framework of complete probability. In order to avoid the optimal value $w$ overfitting, the sparse Bayesian method directly gives a limitation of conditional probability distribution for weight parameters. Here a Gauss condition distribution with zero mean is given by

$$
p(\boldsymbol{w} \mid \boldsymbol{\alpha})=\prod_{i=0}^{N} N\left(w_{i} \mid 0, \alpha_{i}^{-1}\right)
$$

where $\boldsymbol{\alpha}=\left[\alpha_{0}, \alpha_{1}, \ldots, \alpha_{N}\right]$ is a vector of $\mathrm{N}+1$ hyperparameters followed Gamma distributions, this vector $\boldsymbol{\alpha}$ is ultimately responsible for its sparse properties of the model. The likelihood function over the weights is then obtained

$$
\begin{gathered}
p\left(c_{i} \mid \boldsymbol{\alpha}, \quad \sigma^{2}\right)=\left(2 \pi \sigma^{2}\right)^{-\frac{N}{2}} \exp \left[-\frac{1}{2 \sigma^{2}}\|c-\eta w\|^{2}\right] \\
\eta\left(x_{i}\right)=\left[1, k\left(x_{i}, x_{1}\right), \ldots, k\left(x_{i}, x_{n}\right)\right] \\
c_{i}=y\left(x_{i}, \quad \mathrm{w}\right)+\varepsilon_{\mathrm{i}}
\end{gathered}
$$

Set $B=\left[\eta\left(x_{1}\right), \eta\left(x_{2}\right), \ldots, \eta\left(x_{n}\right)\right]$, The function of the RVM can be written as

$$
f(x)=B(x)\left[\sum_{i=1}^{n} a_{i} B\left(x_{i}\right)\right]
$$

Here, the recognition steps of fracture surface image based on the RVM are described as follows

(a) Select the radial basis function (RBF) as the kernel function, the characteristic data in the lowdimensional space is mapped into the high-dimensional space, where the RBF is written as

$$
K(X, Y)=\exp \left(-\frac{\|X-Y\|^{2}}{2 \sigma^{2}}\right), \sigma>0
$$

(b) the parameter $\boldsymbol{\alpha}$ is estimated by multiple iterative optimization

$$
\alpha_{i}^{\text {new }}=\frac{\gamma_{i}}{\mu_{i}^{2}}=\frac{1-\alpha_{i} \Sigma_{i, i}}{\mu_{i}^{2}}
$$

(c) The classified data obtained by Eq.(9) is used to recognize different types of fracture image.

\section{Experimental studies}

Fig. 2 shows several typical fracture images taken by scanning electron microscopy (SEM). From Fig.2, every fracture image has own microstructures. For example, the microstructure of fragile fracture is composed of a series of small crack surfaces (i.e. cleavage plane of each grain) which is similar to the waves in the river. The formation mechanism of dimple fracture is the aggregation of some holes. The intergranular fracture is a fracture formed by grain boundary weakness of metal material along crystal boundary due to improper heat treatment, stress and other factors etc. while observed by the SEM, the intergranular fracture is a polyhedron crystalline structure. Each type of fracture has a unique texture feature. Therefore the different fracture feature extracted effectively and accurately is the key to recognize different metal fracture image.
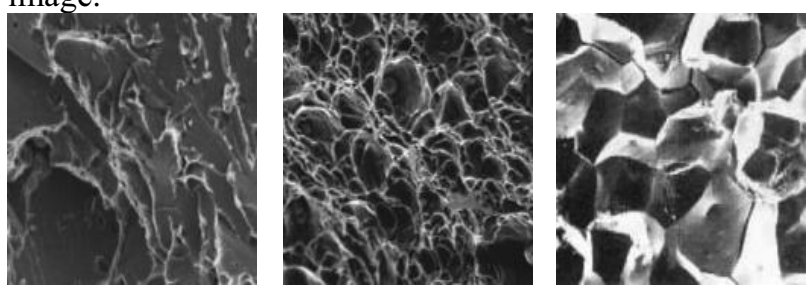

(a)Fragile fracture (b)Dimple fracture (c)Intergranular fracture

Fig.2 Three typical fractures morphology

Here, 100 fragile fracture images, 100 dimple fracture images and 22 intergranular fracture images are selected in the experiment. Each image size is $256 \times 256$. In order to verify the validity of the proposed method, we compared the proposed method with the wavelet entropyRVM and wavelet kurtosis-SVM recognition method.

\section{Comparision between wavelet kurtosis-RVM and wavelet entropy-RVM recognition method.}

Fig.3 illustrate the test results of the wavelet kurtosisRVM and wavelet entropy-RVM recognition method respectively.

From Fig.3(a), in the wavelet kurtosis-RVM recognition, 3 fragile fractures are mistaken as dimple fracture, 3 dimple fractures are mistaken as fragile fracture, and 5 intergranular fractures are mistaken as dimple fracture. The recognition rate of the wavelet kurtosis-RVM recognition method is $90.09 \%$.

From Fig.3(b), in the wavelet entropy-RVM recognition method, 4 fragile fractures are mistaken as dimple fracture, 1 fragile fracture is mistaken as intergranular fracture, 6 dimple fractures are mistaken as fragile fracture, 1 dimple fracture is mistaken as intergranular fracture, and 6 intergranular fracture are mistaken as dimple fracture. The recognition rate of the wavelet entropy-RVM recognition method is $83.78 \%$.

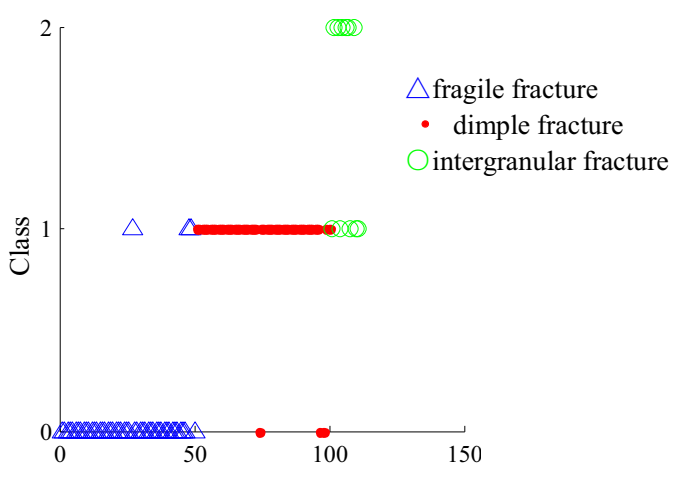

(a). The recognition results (wavelet kurtosis-RVM recognition method) 


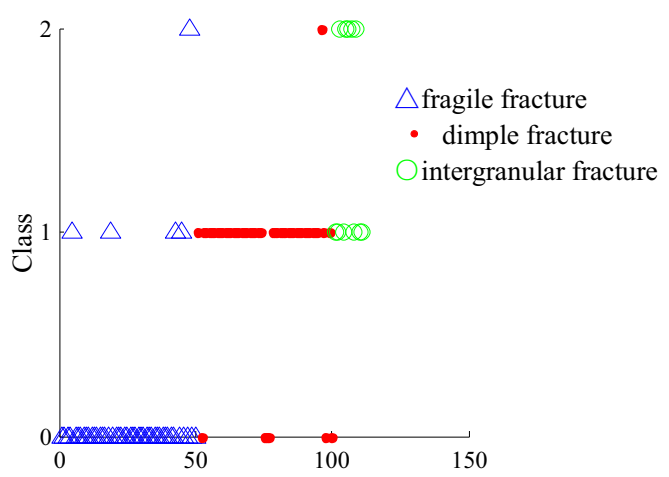

(b). The recognition results (wavelet entropy- recognition method)

Fig3. The recognition results

In the two recognition methods, the classifier is the same, however the feature vector is different, one is the wavelet kurtosis, another is the wavelet entropy. The wavelet entropy is proportional to the square of wavelet coefficients, the wavelet kurtosis is proportional to the 4 th power of wavelet coefficients, obviously, the wavelet kurtosis is more sensitive to the texture feature of metal fracture image than wavelet entropy. The small change of texture feature lead to the significant change of wavelet kurtosis. The wavelet kurtosis is about a hundred times of wavelet entropy. Therefore, the wavelet kurtosis-RVM recognition method is superior to the wavelet entropyRVM recognition method.

\section{Comparison between wavelet kurtosis- RVM and wavelet kurtosis-SVM recognition method.}

In the two recognition methods, i.e. wavelet kurtosisRVM and wavelet kurtosis-SVM, the feature vector is the same, i.e.wavelet kurtosis. However the classifier is difference, one is a RVM, another is a SVM. The recognition results of the two methods are showed in Tab.1.

Tab.1 Comparison of the two recognition methods

\begin{tabular}{lll}
\hline Recognition method & recognition rate $/ \%$ & time $/ \mathrm{s}$ \\
\hline Wavelet kurtosis-RVM & 90.09 & 2.24 \\
Wavelet kurtosis-SVM & 90.09 & 12.68 \\
\hline
\end{tabular}

From Tab.1, the two recognition methods have the satisfactory recognition rate. The recognition rate reaches $90.09 \%$. However, their recognition speed has obvious difference. The training time of the wavelet kurtosisRVM recognition method is far less than that of the wavelet kurtosis-SVM recognition method. In the recognition of 222 fracture images, the training speed in the wavelet kurtosis-RVM is about six times of that in the wavelet kurtosis-SVM recognition method. This is because the number of support vector in the wavelet kurtosis-SVM recognition method linearly increases as the training sample increases. Too many support vectors may cause the possibility of overfitting, the increase of training time and the reduction of recognition speed.

\section{Conclusions}

In this research, a new feature extraction method of metal fracture image based on wavelet kurtosis is proposed. The proposed method is compared with another feature extraction method of metal fracture based on wavelet entropy. At the same time, combining wavelet kurtosis with relevance vector machine, a recognition method of metal fracture image based on wavelet kurtosis-RVM is proposed. The proposed wavelet kurtosis-RVM recognition method is compared with the wavelet kurtosis-SVM recognition method. The experimental results verify the validity of the proposed method. From experimental studies, some valuable conclusions can be obtained

(1) The proposed wavelet kurtosis is proportional to the fourth power of wavelet coefficients. Relative to wavelet entropy, the wavelet kurtosis is more sensitive to the texture change of metal fracture image. Therefore the wavelet kurtosis is more suitable for the feature extraction of fracture image.

(2) The proposed wavelet kurtosis-RVM recognition method is superior to the wavelet entropy-RVM recognition method.

(3) Whether the wavelet kurtosis-RVM recognition method or the wavelet kurtosis-SVM recognition method, the recognition rate is very satisfactory. However their recognition speed has obvious difference. The recognition speed of the former is much faster than that of the latter. Especially with the increase of training sample, this advantage in the wavelet kurtosis-RVM recognition method is more obvious.

\section{Acknowledgements}

The work described in this paper was supported by the Natural Science Foundation of China ((51261024, 51075372) and Key Laboratory of Digital Signal and Image Processing of Guangdong Province (2014GDDSIPL-01).

\section{References}

1. Zhong, Q. P., Zhao, Z. H., Zhang, Z.: 'Develpment of "Fractography" and research of Fracture micromechansim', Journal of Mechanical Strength. 2005, 27, (3), pp. 358-370.

2. Yan, Y. H., Yang, H. L., Wang, C. M.: 'Nonlinear Pattern Recgnition of Metal Fracture Surface Images', Journal of Northeastern University, 2004, 25, (9), pp. 884-886. (In Chinese)

3. Yamagiwa, K., Izumi, S., \& Sakai, S.: 'Detecting method of striation region of fatigue fracture surface using wavelet transform', Journal-Society of Materials Science Japan, 2004, 53, pp. 306-312.

4. $\mathrm{Xu}, \mathrm{F}$. .: 'Feature Extraction and analysis of metal fracture SEM image', (Doctoral dissertation). University of Electronic Science and Technology of China, 2006

5. ZHANG, L., LI, M., YANG, X. Q.: 'Recognition of fracture image based on tree wavelet transform', 
Journal of Nanchang Hangkong University (Natural Science), 2007, 21, (2), pp. 42- 45.(In Chinese)

6. Tipping, M, E.: 'The relevance vector machine'. Proc. of Advances in Neural Information Processing Systems. [S.1.]:MIT Press, 2000, 652-658.

7. Yan, Y. H., Gao, J. H., G, Liu, Y., et al.: 'Recognition and classfication of metal fracture surface models based on wavelet transform', Acta Metallurgica Sinica, 2002, 38, (3), pp. 309-314. (In Chinese)

8. Blanco, S., Figliola, A., Quian Quiroga, R., et al.: 'Time-frequency analysis of electroencephalogram series (III): information transfer function and wavelets packets', Physical Review E, 1998, 57, (1), pp. 932-940.

9. Yordanova, J., Kolev, V., Rosso, O. A., et al. :'Wavelet entropy analysis of event-related potentials indicates modality-independent theta dominance', Journal of Neuroscience Methods, 2002, 117, (1), pp. 99-109.

10. Maisinger, K., Hobson, M. P., Lasenby, A. N.: 'Maximum-entropy image reconstruction using wavelets', Monthly Notices of the Royal Astronomical Society, 2004, 347, (1), pp. 339-354.

11. Li, X., Cui, W., Li, C.:'Research on classification method of wavelet entropy and Fuzzy Neural Networks for motor imagery EEG', Modelling, Identification \& Control (ICMIC), 2012 Proceedings of International Conference on. IEEE, 2012, pp. 478482. 